\title{
Balloon aortic valvuloplasty in pregnancy with severe aortic stenosis and infective endocarditis
}

\author{
Vinotha*, Ruby Jose \\ Department of Obstetrics \& Gynecology, Christian Medical College and Hospital, Vellore-632004, Tamil Nadu, India
}

Received: 3 December 2012

Accepted: 14 December 2012

*Correspondence:

Dr. Vinotha,

E-mail: thomasvinotha@gmail.com

\begin{abstract}
Twenty seven year old lady, previously diagnosed to have aortic stenosis, presented to the obstetric outpatient department at 19 weeks of gestation with fever and breathlessness, NYHA class 4, for one week. Two D Echo revealed left ventricular hypertrophy, a severely stenosed, calcified bicuspid aortic valve, with vegetations on aortic and mitral valves and severe mitral regurgitation. Blood cultures grew nutrionally variant streptococci and she was treated with crystalline penicillin and gentamicin. She stabilised clinically by 21 weeks, by which time, the risk of termination of pregnancy was comparable to continuing the pregnancy. She underwent balloon aortic valvuloplasty. Post balloon aortic valvuloplasty, she was stable. At $34+2$ weeks, she underwent emergency LSCS, the indication being intrauterine growth restriction with fetal compromise and breech presentation. She delivered a baby girl, $1.6 \mathrm{~kg}$, Apgar $9 \& 10$. Our case report highlights the fact that a timely, balloon aortic valvuloplasty can be life saving for patients with pregnancy complicated by severe aortic stenosis and infective endocarditis.
\end{abstract}

Keywords: Pregnancy, Severe aortic stenosis, Balloon aortic valvuloplasty

\section{INTRODUCTION}

Bicuspid aortic valve can present as functionally normal, aortic regurgitation or aortic stenosis, with or without aortic dilatation. During pregnancy, physiological vasodilatation permits regurgitant valvular lesions to be well tolerated. However the physiological increase in preload can complicate valvular stenotic lesions.

\section{CASE REPORT}

Twenty seven year old, gravida 3, para 1, living 1, abortion 1, presented to the obstetric outpatient department at 19 weeks of gestation, with fever and breathlessness, NYHA class 4 for one week.

Three years ago, during her first pregnancy, she was diagnosed to have a bicuspid aortic valve with aortic stenosis. However, she was asymptomatic and her pregnancy was uneventful. She delivered a term baby girl normally, $2.25 \mathrm{~kg}$, who is now alive and well. The second pregnancy ended in a spontaneous miscarriage at $2 \frac{1 / 2}{2}$ months of amenorrhea. Currently, this was her third pregnancy and had been NYHA class 1 until one week before presentation.

On examination there was an ejection systolic murmur heard in the aortic area and a pansystolic murmur heard in the mitral area. Mild bilateral inspiratory crepitations were heard. The uterus was 20 weeks in size. A transthoracic echocardiogram revealed left ventricular hypertrophy, a calcified bicuspid aortic valve with severe aortic stenosis and a small vegetation, severe mitral regurgitation with a larger vegetation, $6 \times 6 \mathrm{~mm}$, attached to the posterior mitral valve leaflet (Figure 1). The aortic peak gradient was $118 \mathrm{mmHg}$ which indicates severe aortic stenosis. Three blood cultures grew nutrionally variant streptococci. She was started on inj. crystalline penicillin and gentamicin according to the culture sensitivity, for a total duration of 4 weeks. As her hemoglobin was $6.7 \mathrm{gm} \%$, she was transfused one pint of blood. By the time she was clinically stable, she was 21 weeks pregnant. The risk of termination of pregnancy at that stage would have been as high as continuing the 
pregnancy, and therefore she was advised to continue the pregnancy.

Hence, as a temporary measure, as the valve appeared mobile on echocardiogram, balloon aortic valvuloplasty was proposed which was accepted by the patient (Figure 2 and 3$)$.

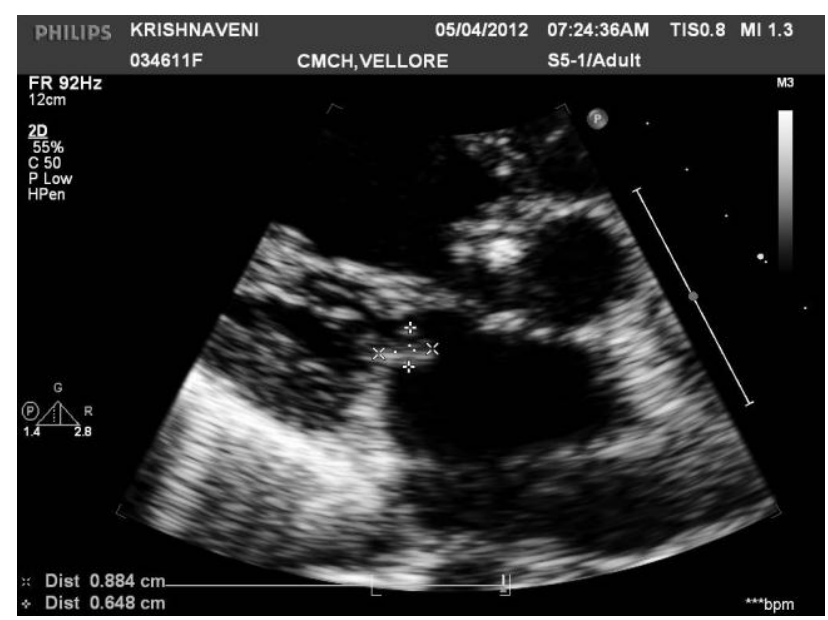

Figure 1: Echo image showing a long axis view of the mitral and aortic valves. There is large vegetation in the mitral valve and a smaller one in the aortic valve.

Post balloon aortic valvuloplasty, angiographically; there was no significant aortic gradient (Figure 2 and 3). There was mild aortic regurgitation visualized angiographically. There was no significant mid cavity obstruction on LV angiogram.

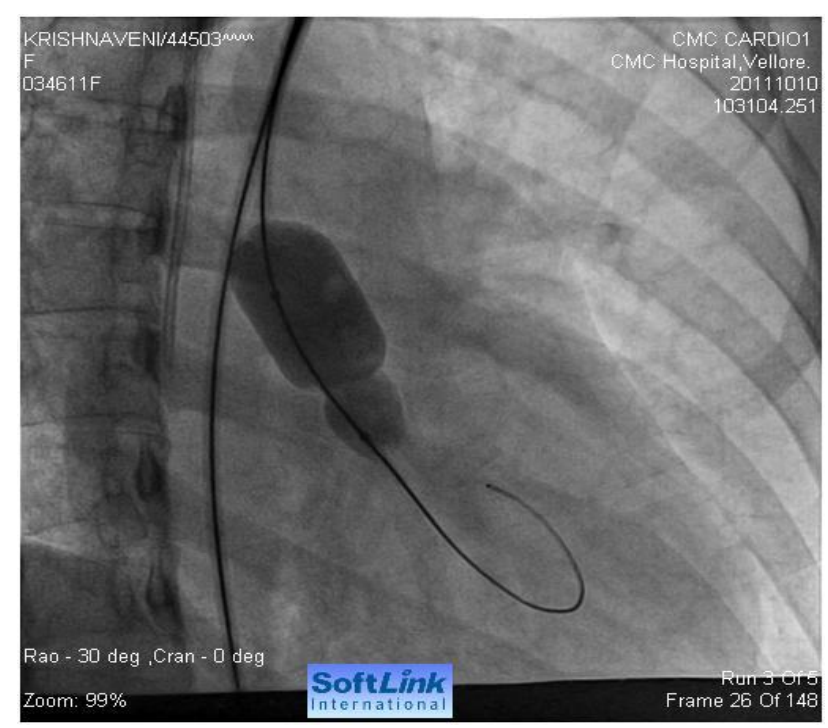

Figure 2: Balloon inflation of the aortic valve. The waist is formed by the narrowed valve.

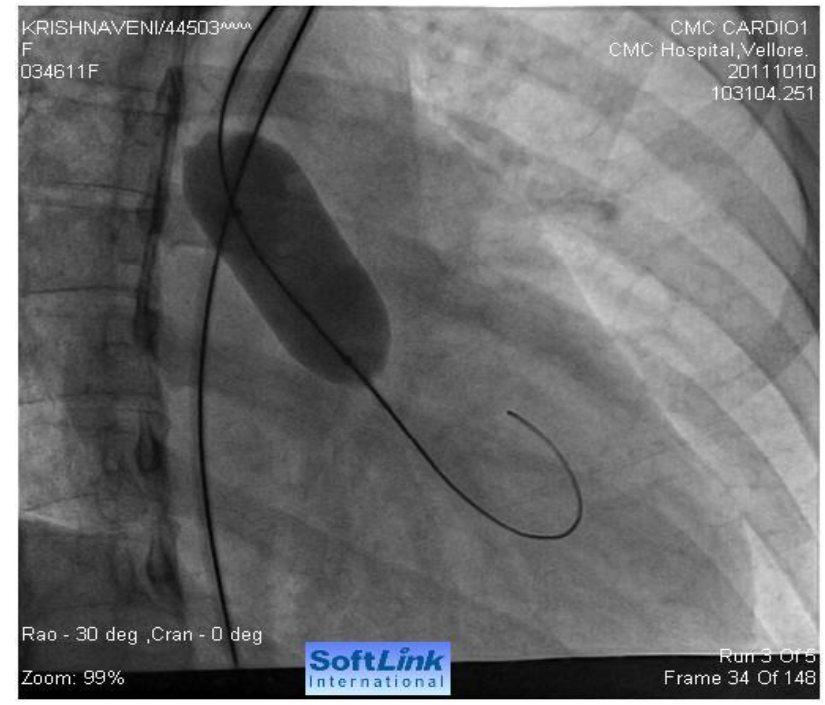

Figure 3: With the balloon inflation, the waist has disappeared as the valve has opened up.

The patient was stable after the procedure. She was discharged after 4 weeks of antibiotics, on oral anticardiac failure measures.

She was followed up regularly in the cardiology and obstetric outpatient department. At 24 weeks, morphology scan revealed no fetal congenital anomalies. At 28 weeks, a baseline scan showed normal fetal wellbeing. At 32 weeks of amenorrhea, she was admitted with concerns of maternal tachycardia despite anticardiac failure measures and intrauterine growth restriction.

With regard to the maternal cardiac status, symptom wise, she was NYHA class 2 but her pulse rate was around 124/minute. Cardiac failure, anaemia and thyroid dysfunction were ruled out. Cardiology opinion was obtained and she was started on tablet hydralazine $25 \mathrm{mg}$ and ivabradine $5 \mathrm{mg}$ twice daily besides tablet digoxin once daily five out of seven days and tablet furosemide. As she had persistent hypokalemia despite oral potassium supplements, furosemide was changed to tablet torsemide $10 \mathrm{mg}$ once daily. With the change in therapy, her pulse rate came down to $90-100 /$ minute and the hypokalemia was corrected.

With regard to the intrauterine growth restriction, the ultrasound scan at 32 weeks, showed an estimated fetal weight of $1.3 \mathrm{~kg}$, with normal amniotic fluid index and normal Dopplers. Fetal surveillance was done with twice weekly with modified biophysical score and Doppler. At $34+2$ weeks, as the baby was in breech presentation and required urgent delivery because of fetal compromise based on fetal surveillance, she underwent emergency caesarean section under general anaesthesia and infective endocarditis prophylaxis. Precautions were taken to maintain fluid balance and to avoid intraoperative 
hypotension. Postoperatively, she was managed in the surgical high dependency unit for 24 hours. She was subsequently shifted to the ward and discharged after suture removal. The postoperative period was otherwise uneventful. The baby girl weighed $1.64 \mathrm{~kg}$ at birth and was transferred to the neonatal intensive care unit for preterm care.

\section{DISCUSSION}

In case of aortic stenosis, the pregnancy related volume expansion, overloads a pressure loaded left ventricle. Though mild and moderate aortic stenosis, when the left ventricular ejection fraction is normal, is tolerated well in pregnancy, delay in conception is recommended in women with symptomatic aortic stenosis and with left ventricular ejection fraction less than $40 \% .^{1}$ In pregnancy, severe aortic stenosis can be complicated by heart failure in $10 \%$ of patients and arrhythmias in $3-25 \%$. $^{2}$

When women with severe aortic stenosis develop major symptoms during pregnancy, case reports suggest that balloon valvuloplasty can reduce the risks of gestation, labor, and delivery and avoid the risks of valve replacement. ${ }^{3}$ However, even in experienced hands, the common post procedural complications are aortic regurgitation and recurrent stenosis which is common within 6 to 12 months. Shielding the gravid uterus from direct radiation and especially shortening fluoroscopic time will minimize radiation exposure. Balloon valvuloplasty can be used as palliative therapy during pregnancy before valve replacement. Our case report highlights the fact that a timely balloon aortic valvuloplasty can be life saving for patients with severe aortic stenosis and pregnancy complicated by infective endocarditis.

\section{ACKNOWLEDGEMENTS}

The authors acknowledge Dr. Jacob Jose, Professor and Head of Cardiology, Unit 2, Christian Medical College and Hospital, Vellore, Tamil Nadu, India and are grateful to him for assisting them in preparing the manuscript and providing technical expertise in providing the echocardiogram and angiogram images.

\section{REFERENCES}

1. Vahanian A, Baumgartner H, Bax J, Butchart E, Dion R, Filippatos G, et al. Guidelines on the management of valvular heart disease: the Task Force on the Management of Valvular Heart Disease of the European Society of Cardiology. Eur Heart J 2007;28:230-68.

2. Regitz-Zagrosek V, Blomstrom Lundqvist $\mathrm{C}$, Borghi C, Cifkova R, Ferreira R, Foidart JM, et al. ESC Guidelines on the management of cardiovascular diseases during pregnancy. Eur Heart J 2011;32:3147-97.

3. Tumelero RT, Duda NT, Tognon AP, Sartori I, Giongo S. Percutaneous Balloon Aortic Valvuloplasty in a Pregnant Adolescent. Arq Bras Cardiol 2003;82:98-101.

DOI: $10.5455 / 2320-1770 . i j \mathrm{rcog} 001812$

Cite this article as: Vinotha, Jose R. Balloon aortic valvuloplasty in pregnancy with severe aortic stenosis and infective endocarditis. Int J Reprod Contracept Obstet Gynecol 2012;1:69-71. 